\title{
The fate of olive oil polyphenols in the gastrointestinal tract: implications of gastric and colonic microflora-dependent biotransformation
}

\author{
Giulia Corona $^{\mathrm{a}, \mathrm{b}}$, Xenofon Tzounis ${ }^{\mathrm{a}}$, M. Assunta Dessi ${ }^{\mathrm{b}}$, Monica Deiana ${ }^{\mathrm{b}}$, Edward S. \\ Debnam $^{c}$, Francesco Visiolid ${ }^{d}$ and Jeremy P. E. Spencer ${ }^{a,{ }^{*}}$ \\ a Molecular Nutrition Group, School of Food Biosciences, University of Reading, Reading RG2 6AP, UK, \\ bDipartimento di Biologia Sperimentale, Sez. Patologia Sperimentale, Università degli Studi di Cagliari, \\ 09042 Monserrato Italy, 'Department of Physiology, Royal Free and University College Medical School, \\ London, UK, dDepartment of Pharmacological Sciences, University of Milan, 20133 Milan, Italy,
}

Running title: Fate of olive oil polyphenols in the Gl tract

${ }^{*}$ Corresponding author:

Dr. Jeremy P.E. Spencer, School of Food Biosciences, University of Reading, PO Box 226, Whiteknights, Reading, RG2 6AP, UK. e-mail: j.p.e.spencer@reading.ac.uk, Telephone: +44 (0) 118378 8724, Fax: +44 (0) 1189310080 


\section{Abstract}

Olive oil is a rich source of polyphenols, in particular the phenolic acids hydroxytyrosol and tyrosol, and their conjugated forms, such as oleuropein. The bioactivity of these compounds in vivo will depend on the extent of their absorption and metabolism. We have conducted a detailed investigation of the absorption, metabolism and microflora-dependent transformation of olive oil polyphenols at different sites in the gastrointestinal tract. The results show that conjugated forms undergo rapid hydrolysis in acidic gastric conditions, resulting in an increase in the amount of free HT and TYR in the small intestine (475\% and 325\%, respectively at 30min). These phenols were demonstrated to be absorbed in both human Caco-2 cells $\left(\mathrm{P}_{\mathrm{app}}=2.18 \pm 0.37 \times 10^{-5}\right.$ for HT and $5.11 \pm 0.59 \times 10^{-5}$ for TYR) and rat segments of the jejunum ( $P_{\text {app }}=1.26 \pm 0.52 \times 10^{-5}$ for $H T$ and $3.55 \pm 0.11 \times 10^{-5}$ for TYR) and the ileum $\left(P_{\text {app }}=1.41 \pm 0.99 \times 10^{-5}\right.$ for HT and $2.91 \pm 0.56 \times 10^{-5}$ for TYR). Upon uptake into jejunum or ileum, the compounds underwent various biotransformations via classic phase I/II metabolism pathways. The major metabolites identified were the $O$ methylated derivatives of $\mathrm{HT}, \mathrm{O}$-glucuronides of $\mathrm{HT}$ and TYR, and a glutathionylated conjugate of HT. In contrast, there was no absorption of oleuropein in either small intestinal model. However oleuropein was rapidly degraded by the colonic microflora resulting in the formation of HT. Our data indicate that although many of the complex polyphenols present in olive oil (oleuropein and secoiridoids derivatives) are not absorbed, these may be degraded in the Gl tract to yield increased amounts of HT and TYR, which may be absorbed and exert biological effects.

Keywords: Olive oil, Hydroxytyrosol, Tyrosol, Absorption, Metabolism, colonic microflora. 


\section{Introduction}

Olive oil is the principal fat component of the Mediterranean diet, and its consumption has been associated with a lower incidence of coronary heart disease (CHD) and certain cancers [1-4]. Extra virgin olive oil contains a variety of simple phenolic compounds, such as hydroxytyrosol (HT), tyrosol (TYR), and their secoiridoid derivatives, such as oleuropein (OL) and its aglycone form. These compounds, together with other components, such as tocopherols, contribute towards the protection of olive oil against auto-oxidation, and have been demonstrated to exert many potentially beneficial biological effects both in vitro and in vivo [5-11]. However, the biological properties of these compounds in vivo will depend on the extent of their absorption and metabolism. In vivo studies have been performed to investigate the bioavailability of olive oil polyphenols by measurement of their excretion in urine following administration [12-15]. It has been reported that hydroxytyrosol and tyrosol levels in urine increase after olive oil administration, and that these compounds were primarily excreted as O-glucuronidated conjugates [15-17]. Hydroxytyrosol may also be $O$-methylated in vivo, and homovanillic acid and homovanillyl alcohol have been measured in human and animal studies in plasma and urine after olive oil ingestion [12, 16, 18, 19]. In addition, human studies conducted in healthy and ileostomy patients fed with polyphenols have show that ingested olive oil phenols were absorbed in healthy and ileostomy subjects, which indicates that most, if not all, are absorbed in the small intestine [20]. However, only few data exist for the absorption and metabolism of olive oil polyphenols in the small intestine, and there are little or no data for their stability in the stomach or their biotransformation in the colon. For example, Manna et al. [21] reported that HT is absorbed by passive diffusion in Caco-2 cells but there are no data on the specific absorption/metabolism of other olive oil polyphenols in the small intestine.

The aim of this study was to investigate the absorption, subsequent biotransformation and potential modification of a range of olive oil polyphenols (including oleuropein and secoiridoid derivatives) in the gastrointestinal tract. In particular we focus on their decomposition in the stomach, their absorption and metabolism in the small intestine and their biotransformation by the microflora present in the large intestine. Knowledge of the detailed biotransformation of olive oil 
polyphenols in the gastrointestinal tract will provide important information regarding potential bioactive components in vivo.

\section{Materials and Methods}

\section{Materials.}

The polyphenolic mixture was obtained by processing extra virgin olive oil by methanol/water extraction as previously described by Caruso et al. [17]. The Caco-2 cell line was obtained from ECACC (Salisbury, Wiltshire UK). Tyrosol and oleuropein were obtained from Extrasynthase (ZI Lyon Nord, Genay, France). Hydroxytyrosol was obtained from Cayman Chemical Company (Ann Arbor MI, USA). $\beta$-Glucuronidase (type L-II from limpets) was purchased from Sigma (Poole, Dorset, UK). Peptone water, yeast extract and bile salts were purchased from Oxoid. Ltd. (Basingstoke, UK). HPLC grade acetonitrile and methanol were purchased from Fisher (Hampton, $\mathrm{NH}$ ) and Elgstat UHP double distilled water (18.2 $\Omega$ grade) was used throughout the study. HPLC columns were purchased from Waters (Watford, Herts, UK). All other reagents used were obtained from Sigma.

\section{Incubation of polyphenolic fraction with simulated gastric juice.}

In order to obtain information about the breakdown susceptibility of conjugated forms, polyphenolic fraction $(1 \mathrm{mg} / \mathrm{ml})$ was submitted to acidic hydrolysis under conditions similar to the gastric environment (water acidified with $\mathrm{HCl}$ to reacha $\mathrm{pH}=2$ ) and incubated at $37^{\circ} \mathrm{C}$ for $30 \mathrm{~min}, 1 \mathrm{~h}, 2 \mathrm{~h}$ and 4h. After incubation, samples were analyzed by HPLC detection (see later).

\section{Transport and metabolism experiments in the perfused rat intestinal model.}

Transport and metabolism experiments were conducted using the in vitro intestinal preparation of Fisher and Gardner $[22,23]$ in which the lumen of isolated intestine is perfused with a segmental flow (defined as perfusion of buffer interspersed with bubbles of the gas mixture) of bicarbonate buffer ( $\mathrm{pH}$ 7.4) equilibrated with $95 \% \mathrm{O}_{2}: 5 \% \mathrm{CO}_{2}$ and containing $28 \mathrm{mM}$ glucose. The bicarbonate 
buffer consisted of $\mathrm{HCO}_{3}{ }^{-} 25 \mathrm{mM}$; Na+ $143 \mathrm{mM}$; $\mathrm{Cl}^{-} 133.7 \mathrm{mM}$; K+ $5.9 \mathrm{mM} ; \mathrm{HPO}^{4-} 1.2 \mathrm{mM}$; $\mathrm{Ca}^{2+} 2$ $\mathrm{mM}$ and $\mathrm{Mg}^{2+} 1.2 \mathrm{mM}$. Male Sprague-Dawley rats $(230-260 \mathrm{~g})$ were anaesthetized with pentobarbital sodium (90 mg/kg, intra-peritoneally) and sections of jejunum $(20-30 \mathrm{~cm}$ long, beginning $10 \mathrm{~cm}$ from the ligament of Treitz) or ileum (20-30 cm long, ending $5 \mathrm{~cm}$ from the ileocaecal junction) were cannulated and the lumen was segmentally perfused with the buffer described above. The intestinal sections were then removed from the animal by stripping them from the mesentery, and suspended in a chamber containing liquid paraffin at $37^{\circ} \mathrm{C}$ whilst maintaining the segmental flow of buffer. Before transport experiments were started the segments were perfused for $40 \mathrm{~min}$ in order to flush blood from the vasculature and to allow fluid absorption to reach a steady state. Thereafter, hydroxytyrosol $(54 \mu \mathrm{M})$, tyrosol $(62,5 \mu \mathrm{M})$, oleuropein $(100 \mu \mathrm{M})$ and polyphenolic fraction $(18 \mathrm{mg} / \mathrm{L})$ were added to the buffer and perfused through the intestinal segment, in a single pass fashion, for up to $80 \mathrm{~min}$. During perfusion, absorbed fluid dropped through the paraffin to the base of the chamber and was collected at timed intervals of $20 \mathrm{~min}$. Samples collected from both the jejunum and the ileum were immediately stored at $-20^{\circ} \mathrm{C}$ until analysis. The presence of glucuronide conjugates in the gut samples was established by treatment with B-glucuronidase. Incubation of samples with enzyme (2000 units $/ \mathrm{ml})$ was for $120 \mathrm{~min}$ at $37^{\circ} \mathrm{C}$ in a $0.1 \mathrm{M}$ sodium phosphate buffer, $\mathrm{pH} 3.8$. The $0.1 \mathrm{M}$ phosphate buffer effectively inhibits all sulphatase activity that the enzyme possesses. A glucuronide of an unknown tyrosol metabolite has been characterized and quantified at $280 \mathrm{~nm}$ with tyrosol as the reference compound.

\section{Cell Culture.}

Sub-cultures of the Caco-2 cells were grown in T-75 culture flasks and passaged with a trypsinversene solution. Cells were cultured in DMEM supplemented with $20 \%$ FBS, $1 \%$ non essential amino acids, $2 \mathrm{mM} \mathrm{L-glutamine,} \mathrm{penicillin} \mathrm{(100} \mathrm{units} / \mathrm{ml})$, and streptomycin $(100 \mathrm{~g} / \mathrm{ml})$ at $37^{\circ} \mathrm{C}$ in $5 \%$ $\mathrm{CO}_{2}$.

\section{Transport experiments in the human Caco-2 cell culture model.}

For all transcellular transport studies, Caco-2 cells were seeded in $12 \mathrm{~mm}$ i.d. Transwell inserts 
(polycarbonate membrane, $0.4 \mu \mathrm{m}$ pore size; Corning Costar Corp.) in 12-well plates at a density of $5 \times 10^{4} \mathrm{cells} / \mathrm{ml}$. The basolateral (serosal) and apical (mucosal) compartments contained 1.5 and 0.5 $\mathrm{ml}$ of culture medium, respectively. Culture medium was replaced three times a week for 21 days. Before the transport experiment media in both chambers was replaced with Hepes-buffered medium (HBM; pH 7.4; $5 \mathrm{mM}$ Hepes, $154 \mathrm{mM} \mathrm{NaCl}$, 4.6mM KCl, 33mM glucose, 5mM $\mathrm{NaHCO}_{3}$, $1.2 \mathrm{mM} \mathrm{Na}_{2} \mathrm{HPO}_{4}$ ). Caco-2 cells in transwells at passage 25 to 40 were used for transport experiments at 21 days post seeding. Only inserts with transepithelial electrical resistance (TEER) values $>300 \Omega / \mathrm{cm}^{2}$ were used for transport experiments. Different concentrations of HT, TYR and OL $(10,50$ and $100 \mu \mathrm{M})$ and POL $(5,25$ and $50 \mu \mathrm{g} / \mathrm{ml})$ in methanol were added in the apical side and upon termination of the $2 \mathrm{~h}$ incubation at $37^{\circ} \mathrm{C}$, samples from both compartments were collected for immediate HPLC analysis.

\section{Synthesis of hydroxytyrosol-glutathione conjugate}

Hydroxytyrosol-glutathione conjugate was prepared by a method similar to that previously described by Spencer et al [24] for catecholamine conjugates, with some modifications. GSH $(10 \mathrm{mM})$ and mushroom tyrosinase (2000 units $/ \mathrm{ml})$ were incubated in sodium acetate buffer (20 $\mathrm{mM}$ ), $\mathrm{pH} 6.5$, at $25^{\circ} \mathrm{C}$ (total volume of $10 \mathrm{ml}$ ) before the drop-wise addition of hydroxytyrosol $(10 \mathrm{mM})$ under constant stirring for $1 \mathrm{~h}$. To stop the reaction the sample mixture was acidified with $0.5 \% \mathrm{HCl}$ $5 \mathrm{M}$ and after centrifugation the supernatant was collected for HPLC analysis.

\section{Colonic microflora fermentation: batch culture.}

The composition of the basal growth medium, per liter, was: peptone $(2 \mathrm{~g})$, yeast extract $(2 \mathrm{~g})$, Tween $80(2 \mathrm{ml})$, hemin $(50 \mathrm{mg})$, vitamin $\mathrm{K} 1(10 \mu \mathrm{l})$, L-cysteine $\mathrm{HCl}(0.5 \mathrm{~g})$, bile salts $(0.5 \mathrm{~g}), \mathrm{NaCl}(0.1 \mathrm{~g})$, NaHCO3 (2g), K2HPO4 (40mg), KH2PO4 (40mg), MgSO4-7H2O (10mg), CaCl2-6H2O (10mg), resazurin $(1 \mathrm{mg})$, and distilled water to volume. The medium was dispensed into the fermentation vessels and autoclaved at $121^{\circ} \mathrm{C}$ for $15 \mathrm{~min}$. Post-autoclaving, the hot medium was purged with oxygen-free nitrogen at the rate $15 \mathrm{ml} / \mathrm{min}$. Fecal slurry of $10 \%(\mathrm{w} / \mathrm{v})$ feces was prepared by homogenizing a stool sample with anaerobic phosphate-buffered saline $(0.1 \mathrm{M}, \mathrm{pH} 7.0)$ in a 
stomacher. The slurry was sieved to remove particulate material. Fecal donors were aged 20 to 40 years, had no history of gastrointestinal disease, and had not taken antibiotics in the 6 months prior to donation. Samples were processed within $10 \mathrm{~min}$ of defecation. The fermentation volume was $150 \mathrm{ml}$. Each batch culture (substrate fermentation and control fermentations) consisted of $90 \%$ basal growth medium and10\% fecal slurry. Oleuropein $(1 \mathrm{mM})$ was added to the vessel and culture $\mathrm{pH}$ was maintained at 6.8-7.0 throughout the incubation. Continuous monitoring of $\mathrm{pH}$ and automatic addition of alkali $(0.5 \mathrm{M} \mathrm{NaOH})$ and acid $(0.5 \mathrm{M} \mathrm{HCl})$ was automated by use of an Electrolab $\mathrm{pH}$ controller. Cultures were incubated at $37^{\circ} \mathrm{C}$ by a heated water jacket, continuously purged with oxygen-free nitrogen $(15 \mathrm{ml} / \mathrm{min})$, and mixed on a magnetic stirrer. The fermenters were made of glass and had sampling ports to remove culture medium for analysis through the incubations, at time $0,4 \mathrm{~h}, 8 \mathrm{~h}, 12 \mathrm{~h}, 24 \mathrm{~h}$ and $48 \mathrm{~h}$. Following sampling from fermentation vessels, the suspension was spun for $5 \mathrm{~min}$ at $13,000 \mathrm{xg}$. The resultant supernatant was used for HPLC analysis. The experiment was carried out in duplicate and using 3 different fecal donors.

\section{HPLC analysis.}

HPLC analysis to measure compound and metabolite concentrations was carried out with a Hewlett-Packard 1100 series liquid chromatograph (Hewlett-Packard, Palo Alto, CA) equipped with a diode array detector linked to the HP ChemStation Software system. Samples were analyzed by reverse-phase HPLC using a Nova-Pak C18 column (4.6 * $250 \mathrm{~mm}$ ) (Waters Company) with $4 \mu \mathrm{m}$ particle size. The temperature of the column was maintained at $30^{\circ} \mathrm{C}$. The mobile phases consisted of a mixture of aqueous methanol $5 \%$ in $0.1 \%$ hydrochloric acid $5 \mathrm{M}(\mathrm{A})$ and a mixture of aqueous acetonitrile $50 \%$ in $0.1 \%$ hydrochloric acid $5 \mathrm{M}(\mathrm{B})$ and were pumped through the column at 0.7 $\mathrm{ml} / \mathrm{min}$. The following gradient system was used (min/\% B): 0/5, 5/5, 40/50, 55/100, 59,9/100, 60/5, whit $10 \mathrm{~min}$ post-run for both compound and metabolite detections. The eluent was monitored by photodiode array detection at $280 \mathrm{~nm}$ and spectra of products obtained over the $200^{\wedge} 600 \mathrm{~nm}$ range. Calibration curves of the compounds were constructed using authentic standards $(0.1$ to $100 \mu \mathrm{M})$ and in each case were found to be linear with correlation coefficients of $>0.995$. 


\section{Calculations and Statistics.}

Apparent permeability coefficients $\left(\mathrm{P}_{\mathrm{app}}\right)$ were calculated with the following equation:

$\mathrm{P}_{\mathrm{app}}=\mathrm{VxdC} / \mathrm{AxC0xdT}=[\mathrm{cm} / \mathrm{sec}]$; where $\mathrm{V}=$ the volume of the solution in the receiving compartment $(\mathrm{ml}), \mathrm{A}=$ the membrane surface area (cell system) or intestinal segment surface area (perfused rat intestinal model), $\mathrm{CO}=$ the initial concentration of compound and $\mathrm{dC} / \mathrm{dt}=$ the change in drug concentration in the receiver solution over time. Intestinal segment surface area was calculated using the equation for the area of a cylinder $(A=2 \pi r i x L i)$, where $L i$ is the length of the perfused segment of intestine and $\mathrm{ri}$ is the internal radius, $0.2 \mathrm{~cm}[25]$. Transport data are expressed as a mean of 3 or more determinations \pm SEM.

\section{Results}

\section{Incubation of polyphenolic fraction with simulated gastric juice.}

Although the gastric conditions in vivo are complex with the food matrix affecting the precise $\mathrm{pH}$, the incubation of polyphenols at gastric $\mathrm{pH}$ can give us information about the stability of polyphenols in the gastric environment. An olive oil polyphenolic fraction $(1 \mathrm{mg} / \mathrm{ml})$ was incubated in water acidified with $\mathrm{HCl}$ to reacha $\mathrm{pH}=2$ at $37^{\circ} \mathrm{C}$ for up to $4 \mathrm{~h}$ to simulate conditions in the stomach, and the products were analyzed by HPLC with photodiode array detection. The results indicate a time dependent hydrolysis of conjugated polyphenols as demonstrated by a decline of compounds represented by peaks with a retention time (RT) between $40 \mathrm{~min}$ and $60 \mathrm{~min}$, and a corresponding progressive increase in peaks representing the hydrolysis products hydroxytyrosol (RT 12,1min) and tyrosol (18,3min $\mathrm{RT}$ ), respectively (figure $1 \mathrm{~A}$ and $1 \mathrm{~B})$. After $30 \mathrm{~min}$ incubation the amount of hydroxytyrosol and tyrosol increased 4.75 and 3.25 times, respectively, and after $4 \mathrm{~h}$ the amount increased 7.41 and 5.15 times, respectively (figure 1C). Interestingly, after 4 hours of hydrolysis, some fractions of the conjugated forms are still present unhydrolyzed. This result indicates that the

amount of hydroxytyrosol and tyrosol that arrive in the small intestine following incubation and 
passage through the acidic conditions of the stomach may be considerably higher than the amount initially present in ingested olive oil.

\section{Transport and metabolism experiments in the perfused rat intestinal model.}

The comparative absorption of HT, TYR, OL and polyphenolic fraction, and the extents to which they are conjugated and metabolized during transfer across jejunum and ileum to the serosal side was investigated. The transferred compounds were identified and quantified by HPLC with diode array detection. Fig 2 illustrates the time course for transfer and metabolism of HT (fig. 2A and B), TYR (fig.2C and D), and the polyphenolic extract (POL) (fig.2E and F) in jejunum and ileum respectively. Interestingly, oleuropein was not transferred across either small intestinal segments, at any time point. In contrast, hydroxytyrosol (fig. 2A and B) and tyrosol (fig. 2C and D) were rapidly absorbed in both jejunum and ileum, following perfusion with the single phenolics, and significant amounts of phase I and phase II metabolites were found in the serosal fluid. After HT perfusion, HT, 3-O-methyl-hydroxytyrosol (homovanillyl alcohol) and the respective glucuronides of both compounds were detected following jejunum and ileum passage (fig. 2A and B). Similarly, after perfusion with tyrosol, the parental compound and two new peaks were observed in the serosal fluid of both gut segments (fig. $2 \mathrm{C}$ and D). Both new peaks disappeared after glucuronidase treatment, with a subsequent increase in tyrosol and a new peak, indicating that a glucuronide of tyrosol and another glucuronide metabolite were present. This new peak had similar spectral properties as tyrosol, but it remains to be identified. Figure 3 indicates that both HT and TYR are absorbed in the parental form to a greater extent in the ileum than in the jejunum (fig. $3 \mathrm{~A}$ and $\mathrm{B}$ ), which may reflect enzymatic profiles in these tissues. The perfusion of the polyphenolic fraction also resulted in the transfer of compounds, tyrosol and hydroxytyrosol, and lead to the formation of glucuronide conjugates and homovanillyl alcohol (fig. 2E and F and fig. 3C). HPLC chromatograms did not reveal the presence of other metabolite resulting from the polyphenolic extract perfusion. We calculated the apparent permeability coefficients for hydroxytyrosol and tyrosol in both jejunum

$\left(\mathrm{P}_{\mathrm{ap}}[\mathrm{HT}]=1.26 \pm 0.52 \times 10^{-5} ; \mathrm{P}_{\mathrm{app}}[\mathrm{TYR}]=3.55 \pm 0.11 \times 10^{-5}\right)$ and ileum $\left(\mathrm{P}_{\mathrm{app}}[\mathrm{HT}]=1.41 \pm 0.99 \times 10^{-5} ; \mathrm{P}_{\mathrm{app}}\right.$ 
$[T Y R]=2.91 \pm 0.56 \times 10^{-5}$ ) (figure 4), indicating that they are well absorbed in both segments of small intestine.

\section{Transport experiments in Caco-2 model.}

Analysis by HPLC of apical (AP) and basolateral (BA) medium over a $2 \mathrm{~h}$ incubation period with HT and TYR demonstrated the enterocyte-mediated absorption and metabolism of the phenolic compounds investigated. Figure 5 (panel A) shows a typical HPLC chromatogram obtained after analysis of medium from the BA side, following a $2 \mathrm{~h}$ incubation with $\mathrm{HT}$. It was observed that the HT concentration decreases on the AP side, and that the compound can be recovered from the BA side, thus demonstrating transfer. HPLC chromatograms also show the presence of two new peaks (RT 16.18 and $21.78 \mathrm{~min}$ ), on both, AP and BA side. The peak at 21.78min has been identified by RT and spectra comparison with authentic standards as homovanillyl alcohol, the 3-O-methylated metabolite of $\mathrm{HT}$. The peak at $16.18 \mathrm{~min}$ was identified as a glutathionyl conjugate of $\mathrm{HT}$, by comparison with a synthesized standard of HT-GSH (see methods) (figure 5B). In comparison to experiments with the isolated small intestinal model that demonstrated HT glucuronidation, using the caco-2 model the majority of the HT (90\%) appeared on the basolateral side as un-metabolized HT (figure 3D), and no glucuronidated conjugates were observed. When Caco-2 monolayers were incubated with TYR, we observed a decreasing amount of TYR in the AP, and we recovered the compound in the BA. In this case we did not detect any new metabolite peaks in the chromatograms. In agreement with rat small intestinal studies, when Caco-2 cells where incubated with oleuropein, we did not measure any significant decrease of compound on the AP side, and the compound was not detected on the BA side at any concentration.

Following incubation of the monolayer with the polyphenolic fraction, (a mixture containing HT and TYR), we measured a decrease in both TYR and HT on the AP side, and both compounds were detected on the BA side. No further peaks were detected on the BA side, indicating that other compounds present in the polyphenolic mixture were not transferred across the monolayer.

The rate of absorption (figure 6) and apparent permeability coefficients $\left(\mathrm{P}_{\text {app }}\right)$ (figure 4) were calculated in order to estimate the bioavailability of the compounds. TYR absorption rate was 
about $60 \%$ for all the concentrations loaded, and HT absorption increased with the concentration, and was between $35 \%$ and $58 \%$ (figure 4 ). The $P_{\text {app }}$ coefficients calculated $\left(2.18 \pm 0.37 \times 10^{-5}\right.$ and $5.11 \pm 0.59 \times 10^{-5} \mathrm{~cm} / \mathrm{sec}$ for $\mathrm{HT}$ and TYR respectively) suggest that HT and TYR are well absorbed in the small intestine following their oral ingestion. Previous studies have positively correlated $\mathrm{P}_{\text {app }}$ coefficients obtained on Caco-2 cells, with the absorption in humans, indicating $100 \%$ absorption for values $>1{ }^{*} 10^{-6}[26]$. Therefore, we propose that both HT and TYR are very well absorbed in the small intestine.

\section{Colonic microflora fermentation: batch culture}

Previous studies have shown that oleuropein is stable in human gastric juice and duodenal fluid [20]. Based on the fact that our data demonstrate that oleuropein is not absorbed or metabolized in the small intestine, it is likely to reach the large intestine where it will be subject to degradation by the colonic microflora. To gain insight into the colonic microflora-mediated biotransformations we utilized a anaerobic fermentation system. Anaerobic suspensions of human colonic micro flora were incubated with $\mathrm{OL}$, the supernatants were collected at various time points and analyzed by HPLC in order to characterize and to quantify OL and OL-derived microfloral metabolites. A representative HPLC chromatogram of the ferment is shown in figure 7 (panel A). The results demonstrate that oleuropein was quickly and extensively degraded resulting in the formation of 3 compounds detected at $11.6 \mathrm{~min}, 36.8 \mathrm{~min}$ and $53.9 \mathrm{~min} \mathrm{RT}$ (fig.7A). Based on the RT, the UV spectrum, and confirmed by comparisons with an authentic standard, the peak at $11.6 \mathrm{~min}$ was identified as hydroxytyrosol. The two other metabolites remain tp be identified. Figure 7B depicts the time-dependent biotransformation of oleuropein by colonic microflora cultures that were established from samples obtained from in 3 individual human donors. In agreement with previous work on the colonic micro flora-catalyzed degradation of other polyphenols [27], a comparison of the different metabolite profiles, as shown in panels B1, B2, and B3 of figure 7, demonstrates that the biotransformation of oleuropein was greatly influenced by individual variations in the microbial composition, However, although the time course for different donors varies (depending on the variation on microflora composition), the micro flora of all donors fully degraded oleuropein 
resulting in the appearance of 3 new metabolites, one of which being HT. Thus, as previously demonstrated for various phenolic acids [28, 29], the micro flora-dependent hydrolysis of oleuropein may consequently increase the bioavailability oleuropein-derived HT via the uptake of HT through the large intestine.

\section{Discussion}

Despite the substantial body of evidence linking the in vitro properties of olive oil polyphenols to the health benefits associated with olive oil consumption, there are limited data on absorption and metabolism. In order to obtain more detailed information about their mechanism of action in vivo, it is essential to know in which form olive oil polyphenols are when they reach the circulation and ultimately the tissues. Presently, the vast majority of information in the literature is based on analysis of blood and urine samples in animal and human studies [12-14, 16, 17, 30-33]. However, these studies do not differentiate between the contribution of gastrointestinal metabolism and overall absorption and biotransformation of olive oil polyphenols. We observed significant nonenzymatic hydrolysis of the conjugated forms olive oil polyphenols under conditions similar to those expected during transit through the stomach in vivo. Although conditions in vivo are more complex than in the model used here, with the food matrix affecting the precise $\mathrm{pH}$, it is highly likely that the gastric environment achieves a $\mathrm{pH}$ of at least 2-3 for a period around 30-minutes, i.e. in order that sufficient enzymatic protein degradation may occur (pepsin). Therefore, we suggest that under such conditions the stability of complex olive oil polyphenols may be affected and could result in a significant increase in the amount of HT and TYR that arrives in the small intestine (4.75 and 3.25 times respectively after $30 \mathrm{~min}$ ) via the pyloroduodenal junction. In this case, higher amounts of HT and TYR may be presented for absorption in the jejunum and ileum than would be expected.

Our absorption studies agree with previous investigations which have shown the presence of HT and TYR glucuronides in urine following ingestion of olive oil polyphenols [12, 13, 17, 31, 33]. We 
detected HT, TYR, homovanillyl alcohol and their glucuronides in the rat small intestine model, which agreed with experiments performed in Caco-2 cells, except that no glucuronides were detected in the cellular model. This is presumably because Caco-2 cells do not possess UDPglucuronosyl transferase activity due to their colonic origin [34]. We stress that caution should be used when interpreting absorption and metabolism data from this cell model as it could underestimate the true level of absorption of a polyphenol, especially that of the glucuronide conjugates. Similarly, use of the isolated rat small intestine model may not generate other potential human metabolites. For example, the results obtained with Caco-2 cells for hydroxytyrosol show that the compound is also glutathione conjugated, which is not the case in the rat small intestinal segment model. As mentioned, these differences reflect different characteristics and enzymatic profile which exist between Caco-2 cells and small intestine segments, and need to be considered when interpreting all polyphenol absorption data. Indeed the Caco-2 model has been considered a suitable model for intestinal CYP3A4 mediated first pass metabolism, but not necessarily for UGT mediated glucuronidation $[35,36]$. It should also be noted that not all intestinal drug metabolizing enzymes are present in this cell line, even though Caco-2 cells have been extensively used in studies of uptake and metabolism of flavonoids [21, 37, 38].

Data from the two models give us a more complete understanding of how these compounds are absorbed and metabolized in humans. We have identified for the first time a glutathione conjugate of hydroxytyrosol generated in Caco-2 cells. This metabolite may be formed either enzymatically via the action of glutathione S-transferase or non-enzymatically via oxidative metabolism of HT followed by its reaction with GSH [39]. Conjugations with thiols, such as glutathione, represent a major target for quinones, and the detoxification of quinones by GSH is generally considered to be cytoprotective. In addition, thiol conjugates of phenolics may be major bioactive forms in vivo and may contribute to positive health effects. We show that the majority of HT and TYR is absorbed, and underwent O-methylation (HT), glucuronidation (HT and TYR) and glutathionyl conjugation (HT) in the small intestine. O-methylation and glucuronidation process are common metabolic pathways in the small intestine for plant derived phenolic acids [23] and in agreement with previous 
studies $[12,16,19]$ showing the presence of these metabolites in plasma and urine after oral administration. Furthermore, in our experiments we did not reveal the presence of homovanillic acid, 3-hydroxy-4-methoxy phenylacetic acid, 2-(3,4-dimethoxyphenyl)-ethanol and sulfateconjugate metabolites, hydroxytyrosol metabolites found in rat urine [19], so we can presume that these metabolites are formed after transport across the small intestine, most probably in the liver.

Oleuropein was not absorbed in the parental form in the small intestine and was not degraded under acidic conditions. Consequently it is likely to reach the large intestine, where it may be subjected to rapid degradation by the colonic microflora. Three major degradation products were produced by the microflora, one of them identified as being HT. Metabolism by colonic bacterial species may have the effect of increasing overall HT bioavailability, as released HT can be absorbed in the colon, as has been shown for other phenolic acids [28, 29]. In addition, oleuropein itself may possess potential prebiotic properties if bacterial groups, such as Bifidobacteria and Lactobacillus, may utilize it as a carbon source, but others such as Clostridium and E. coli cannot. Due to the rapid rate of oleuropein fermentation in the gut model, future investigations are planned to investigate whether oleuropein fermentation by colonic microflora can result in potential 'prebiotic' changes in microbial composition.

Although metabolic processes in the small intestine may act to decrease the bioavailability and consequently the biological effects of native polyphenols, such as hydroxytyrosol and tyrosol, their resultant metabolites may be capable of exerting biological effects in vivo: For example Tuck and al. [19] reported that the radical scavenging potency of homovanillyl alcohol was similar to hydroxytyrosol and that of the 3-O-glucuronide conjugate was more potent when screened in an in vitro assay system. Whilst there has been a major focus on the antioxidant properties, there is an emerging evidence that plant derived polyphenols, in particular flavonoids, and their in vivo metabolites can also modulate the activity of specific proteins involved in intracellular signaling pathways [40]. Many investigations indicate that flavonoids may interact within the mitogenactivated protein kinase (MAP kinase) signaling pathway [41, 42] and it is possible that through 
oxidative mechanisms phenolic acids may act in the same way. As hydroxytyrosol, tyrosol and their metabolites are relatively well absorbed in the small intestine compared to flavonoids, future investigations will center on their potential cellular actions, as has been shown for other plant derived polyphenol metabolites [39, 41, 43-45].

Our study provides additional information regarding the breakdown of complex olive oil polyphenols in the Gl tract, in particular the stomach and the large intestine. We provide novel evidence for the breakdown of complex secoirids derivatives of olive oil polyphenols in a physiological time frame in the stomach. This gastric biotransformation effectively increases the relative amount of hydroxytyrosol and tyrosol entering the small intestine. We also identify a new metabolite of hydroxytyrosol (glutathione conjugated) and highlight the importance of comparing the transfer and metabolism of polyphenols in two different models of the small intestine. Finally, we show for the first time that although oleuropein (a major olive oil polyphenol) is not absorbed in the small intestine it is rapidly degraded by the colonic microflora to yield hydroxytyrosol, which may then be absorbed.

Acknowledgements: This work was supported by PIC-Interreg III, the Medical Research Council (G0400278/NI02) and the BBSRC (grants: BB/C518222/1 and 45/D15524). We also thank Dr. J. Vulevic and Prof. G. Gibson for their help with the colonic metabolism studies. 


\section{References}

[1] Braga, C., La Vecchia, C., Franceschi, S., Negri, E., Parpinel, M., Decarli, A., Giacosa, A., and Trichopoulos, D. (1998) "Olive oil, other seasoning fats, and the risk of colorectal carcinoma", Cancer 82, 448-453.

[2] Keys, A. (1995) "Mediterranean diet and public health: personal reflections", Am J Clin Nutr 61, 1321S-1323S.

[3] Keys, A., Menotti, A., Karvonen, M.J., Aravanis, C., Blackburn, H., Buzina, R., Djordjevic, B.S., Dontas, A.S., Fidanza, F., Keys, M.H., and et al. (1986) "The diet and 15-year death rate in the seven countries study", Am J Epidemiol 124, 903-915.

[4] Owen, R.W., Haubner, R., Wurtele, G., Hull, E., Spiegelhalder, B., and Bartsch, H. (2004) "Olives and olive oil in cancer prevention", Eur J Cancer Prev 13, 319326.

[5] de la Puerta, R., Ruiz Gutierrez, V., and Hoult, J.R. (1999) "Inhibition of leukocyte 5-lipoxygenase by phenolics from virgin olive oil", Biochem Pharmacol 57, 445-449.

[6] Deiana, M., Aruoma, O.I., Bianchi, M.L., Spencer, J.P., Kaur, H., Halliwell, B., Aeschbach, R., Banni, S., Dessi, M.A., and Corongiu, F.P. (1999) "Inhibition of peroxynitrite dependent DNA base modification and tyrosine nitration by the extra virgin olive oil-derived antioxidant hydroxytyrosol", Free Radic Biol Med 26, $762-769$. 
[7] Manna, C., Galletti, P., Cucciolla, V., Montedoro, G., and Zappia, V. (1999) "Olive oil hydroxytyrosol protects human erythrocytes against oxidative damages", J Nutr Biochem 10, 159-165.

[8] Marrugat, J., Covas, M.I., Fito, M., Schroder, H., Miro-Casas, E., Gimeno, E., Lopez-Sabater, M.C., de la Torre, R., and Farre, M. (2004) "Effects of differing phenolic content in dietary olive oils on lipids and LDL oxidation--a randomized controlled trial", Eur J Nutr 43, 140-147.

[9] Salami, M., Galli, C., De Angelis, L., and Visioli, F. (1995) "Formation of F2isoprostanes in oxidized low density lipoprotein: inhibitory effect of hydroxytyrosol", Pharmacol Res 31, 275-279.

[10] Visioli, F., Caruso, D., Galli, C., Viappiani, S., Galli, G., and Sala, A. (2000) "Olive oils rich in natural catecholic phenols decrease isoprostane excretion in humans", Biochem Biophys Res Commun 278, 797-799.

[11] Visioli, F. and Galli, C. (1994) "Oleuropein protects low density lipoprotein from oxidation", Life Sci 55, 1965-1971.

[12] Miro-Casas, E., Covas, M.I., Farre, M., Fito, M., Ortuno, J., Weinbrenner, T., Roset, P., and de la Torre, R. (2003) "Hydroxytyrosol disposition in humans", Clin Chem 49, 945-952.

[13] Miro-Casas, E., Covas, M.I., Fito, M., Farre-Albadalejo, M., Marrugat, J., and de la Torre, R. (2003) "Tyrosol and hydroxytyrosol are absorbed from moderate and sustained doses of virgin olive oil in humans", Eur J Clin Nutr 57, 186-190.

[14] Miro-Casas, E., Farre Albaladejo, M., Covas, M.I., Rodriguez, J.O., Menoyo Colomer, E., Lamuela Raventos, R.M., and de la Torre, R. (2001) "Capillary gas 
chromatography-mass spectrometry quantitative determination of hydroxytyrosol and tyrosol in human urine after olive oil intake", Anal Biochem 294, 63-72.

[15] Visioli, F., Galli, C., Bornet, F., Mattei, A., Patelli, R., Galli, G., and Caruso, D. (2000) "Olive oil phenolics are dose-dependently absorbed in humans", FEBS Lett 468, 159-160.

[16] Visioli, F., Galli, C., Grande, S., Colonnelli, K., Patelli, C., Galli, G., and Caruso, D. (2003) "Hydroxytyrosol excretion differs between rats and humans and depends on the vehicle of administration", J Nutr 133, 2612-2615.

[17] Caruso, D., Visioli, F., Patelli, R., Galli, C., and Galli, G. (2001) "Urinary excretion of olive oil phenols and their metabolites in humans", Metabolism 50, $1426-1428$.

[18] D'Angelo, S., Manna, C., Migliardi, V., Mazzoni, O., Morrica, P., Capasso, G., Pontoni, G., Galletti, P., and Zappia, V. (2001) "Pharmacokinetics and metabolism of hydroxytyrosol, a natural antioxidant from olive oil", Drug Metab Dispos 29, 1492-1498.

[19] Tuck, K.L., Hayball, P.J., and Stupans, I. (2002) "Structural characterization of the metabolites of hydroxytyrosol, the principal phenolic component in olive oil, in rats", J Agric Food Chem 50, 2404-2409.

[20] Vissers, M.N., Zock, P.L., Roodenburg, A.J., Leenen, R., and Katan, M.B. (2002) "Olive oil phenols are absorbed in humans", J Nutr 132, 409-417.

[21] Manna, C., Galletti, P., Maisto, G., Cucciolla, V., D'Angelo, S., and Zappia, V. (2000) "Transport mechanism and metabolism of olive oil hydroxytyrosol in Caco-2 cells", FEBS Lett 470, 341-344. 
[22] Fisher, R.B. and Gardner, M.L. (1974) "A kinetic approach to the study of absorption of solutes by isolated perfused small intestine", J Physiol 241, 211234.

[23] Spencer, J.P., Chowrimootoo, G., Choudhury, R., Debnam, E.S., Srai, S.K., and Rice-Evans, C. (1999) "The small intestine can both absorb and glucuronidate luminal flavonoids", FEBS Lett 458, 224-230.

[24] Spencer, J.P., Jenner, P., Daniel, S.E., Lees, A.J., Marsden, D.C., and Halliwell, B. (1998) "Conjugates of catecholamines with cysteine and GSH in Parkinson's disease: possible mechanisms of formation involving reactive oxygen species", $J$ Neurochem 71, 2112-2122.

[25] Kim, M. (1996) "Absorption of polyethylene glycol oligomers (330-1 122 Da) is greater in the jejunum than in the ileum of rats", J Nutr 126, 2172-2178.

[26] Artursson, P. and Karlsson, J. (1991) "Correlation between oral drug absorption in humans and apparent drug permeability coefficients in human intestinal epithelial (Caco-2) cells", Biochem Biophys Res Commun 175, 880-885.

[27] Rechner, A.R., Smith, M.A., Kuhnle, G., Gibson, G.R., Debnam, E.S., Srai, S.K., Moore, K.P., and Rice-Evans, C.A. (2004) "Colonic metabolism of dietary polyphenols: influence of structure on microbial fermentation products", Free Radic Biol Med 36, 212-225.

[28] Rios, L.Y., Gonthier, M.P., Remesy, C., Mila, I., Lapierre, C., Lazarus, S.A., Williamson, G., and Scalbert, A. (2003) "Chocolate intake increases urinary excretion of polyphenol-derived phenolic acids in healthy human subjects", Am J Clin Nutr 77, 912-918. 
[29] Rechner, A.R., Kuhnle, G., Hu, H., Roedig-Penman, A., van den Braak, M.H., Moore, K.P., and Rice-Evans, C.A. (2002) "The metabolism of dietary polyphenols and the relevance to circulating levels of conjugated metabolites", Free Radic Res 36, 1229-1241.

[30] Covas, M.I., Miro-Casas, E., Fito, M., Farre-Albadalejo, M., Gimeno, E., Marrugat, J., and De La Torre, R. (2003) "Bioavailability of tyrosol, an antioxidant phenolic compound present in wine and olive oil, in humans", Drugs Exp Clin Res 29, 203-206.

[31] Miro Casas, E., Farre Albadalejo, M., Covas Planells, M.I., Fito Colomer, M., Lamuela Raventos, R.M., and de la Torre Fornell, R. (2001) "Tyrosol bioavailability in humans after ingestion of virgin olive oil", Clin Chem 47, 341343.

[32] Tuck, K.L., Freeman, M.P., Hayball, P.J., Stretch, G.L., and Stupans, I. (2001) "The in vivo fate of hydroxytyrosol and tyrosol, antioxidant phenolic constituents of olive oil, after intravenous and oral dosing of labeled compounds to rats", $J$ Nutr 131, 1993-1996.

[33] Visioli, F., Caruso, D., Plasmati, E., Patelli, R., Mulinacci, N., Romani, A., Galli, G., and Galli, C. (2001) "Hydroxytyrosol, as a component of olive mill waste water, is dose- dependently absorbed and increases the antioxidant capacity of rat plasma", Free Radic Res 34, 301-305.

[34] Le Ferrec, E., Chesne, C., Artusson, P., Brayden, D., Fabre, G., Gires, P., Guillou, F., Rousset, M., Rubas, W., and Scarino, M.L. (2001) "In vitro models of the intestinal barrier. The report and recommendations of ECVAM Workshop 46. 
European Centre for the Validation of Alternative methods", Altern Lab Anim 29, 649-668.

[35] Fisher, M.B., Paine, M.F., Strelevitz, T.J., and Wrighton, S.A. (2001) "The role of hepatic and extrahepatic UDP-glucuronosyltransferases in human drug metabolism", Drug Metab Rev 33, 273-297.

[36] Gregory, P.A., Lewinsky, R.H., Gardner-Stephen, D.A., and Mackenzie, P.I. (2004) "Regulation of UDP glucuronosyltransferases in the gastrointestinal tract", Toxicol Appl Pharmacol 199, 354-363.

[37] Walgren, R.A., Lin, J.T., Kinne, R.K., and Walle, T. (2000) "Cellular uptake of dietary flavonoid quercetin 4'-beta-glucoside by sodium-dependent glucose transporter SGLT1", J Pharmacol Exp Ther 294, 837-843.

[38] Walgren, R.A., Walle, U.K., and Walle, T. (1998) "Transport of quercetin and its glucosides across human intestinal epithelial Caco-2 cells", Biochem Pharmacol 55, 1721-1727.

[39] Spencer, J.P., Kuhnle, G.G., Williams, R.J., and Rice-Evans, C. (2003) "Intracellular metabolism and bioactivity of quercetin and its in vivo metabolites", Biochem J 372, 173-181.

[40] Williams, R.J., Spencer, J.P., and Rice-Evans, C. (2004) "Flavonoids: antioxidants or signalling molecules?" Free Radic Biol Med 36, 838-849.

[41] Spencer, J.P., Rice-Evans, C., and Williams, R.J. (2003) "Modulation of prosurvival Akt/protein kinase B and ERK1/2 signaling cascades by quercetin and its in vivo metabolites underlie their action on neuronal viability", J Biol Chem 278, 34783-34793. 
[42] Spencer, J.P. and Murphy, K.P. (2002) "Activation of cyclic AMP-dependent protein kinase is required for long-term enhancement at corticostriatal synapses in rats", Neurosci Lett 329, 217-221.

[43] Spencer, J.P., Abd-el-Mohsen, M.M., and Rice-Evans, C. (2004) "Cellular uptake and metabolism of flavonoids and their metabolites: implications for their bioactivity", Arch Biochem Biophys 423, 148-161.

[44] Spencer, J.P., Schroeter, H., Crossthwaithe, A.J., Kuhnle, G., Williams, R.J., and Rice-Evans, C. (2001) "Contrasting influences of glucuronidation and Omethylation of epicatechin on hydrogen peroxide-induced cell death in neurons and fibroblasts", Free Radic Biol Med 31, 1139-1146.

[45] Spencer, J.P., Schroeter, H., Kuhnle, G., Srai, S.K., Tyrrell, R.M., Hahn, U., and Rice-Evans, C. (2001) "Epicatechin and its in vivo metabolite, 3'-O-methyl epicatechin, protect human fibroblasts from oxidative-stress-induced cell death involving caspase-3 activation", Biochem J 354, 493-500. 
Figure 1
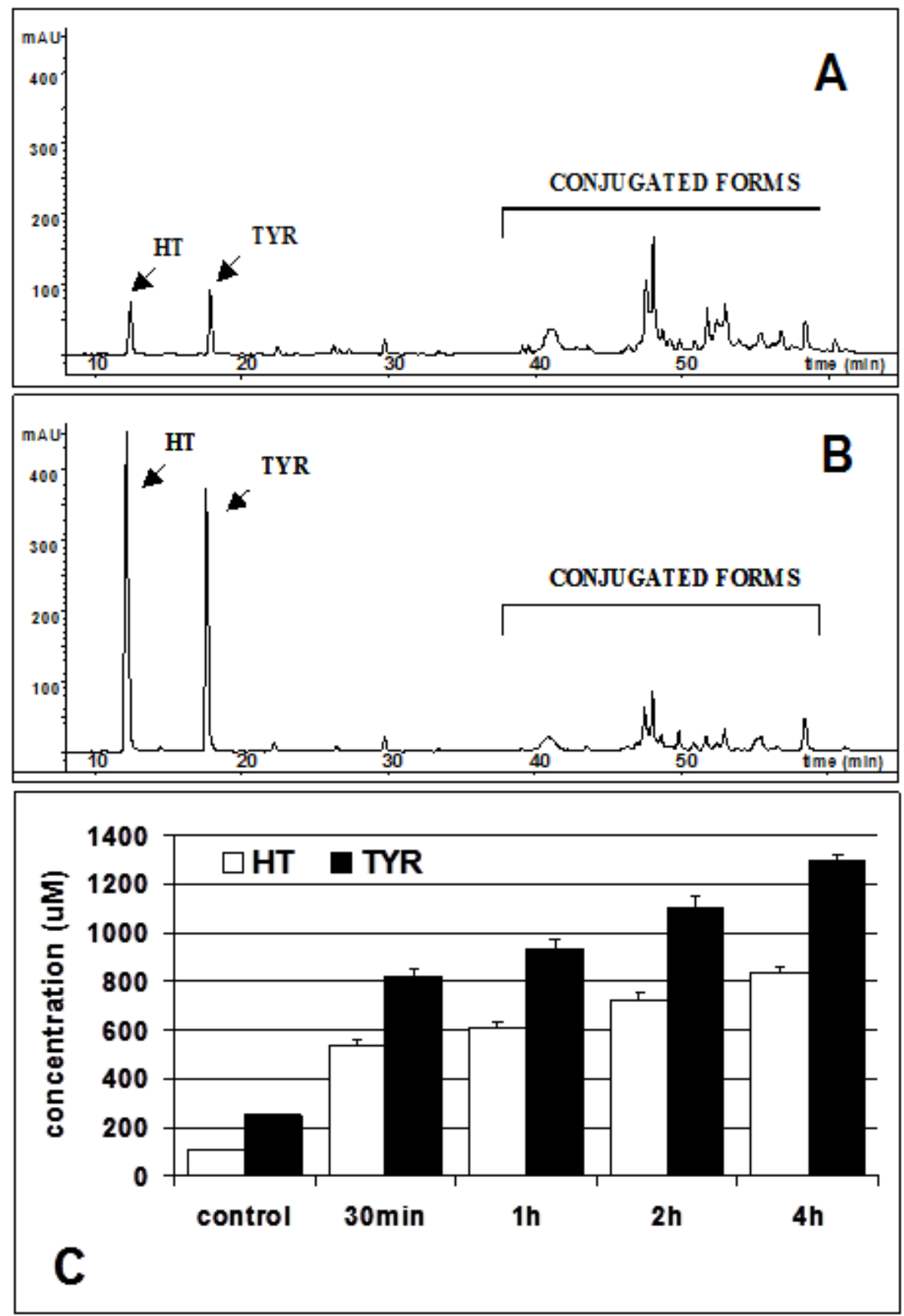
Figure 2
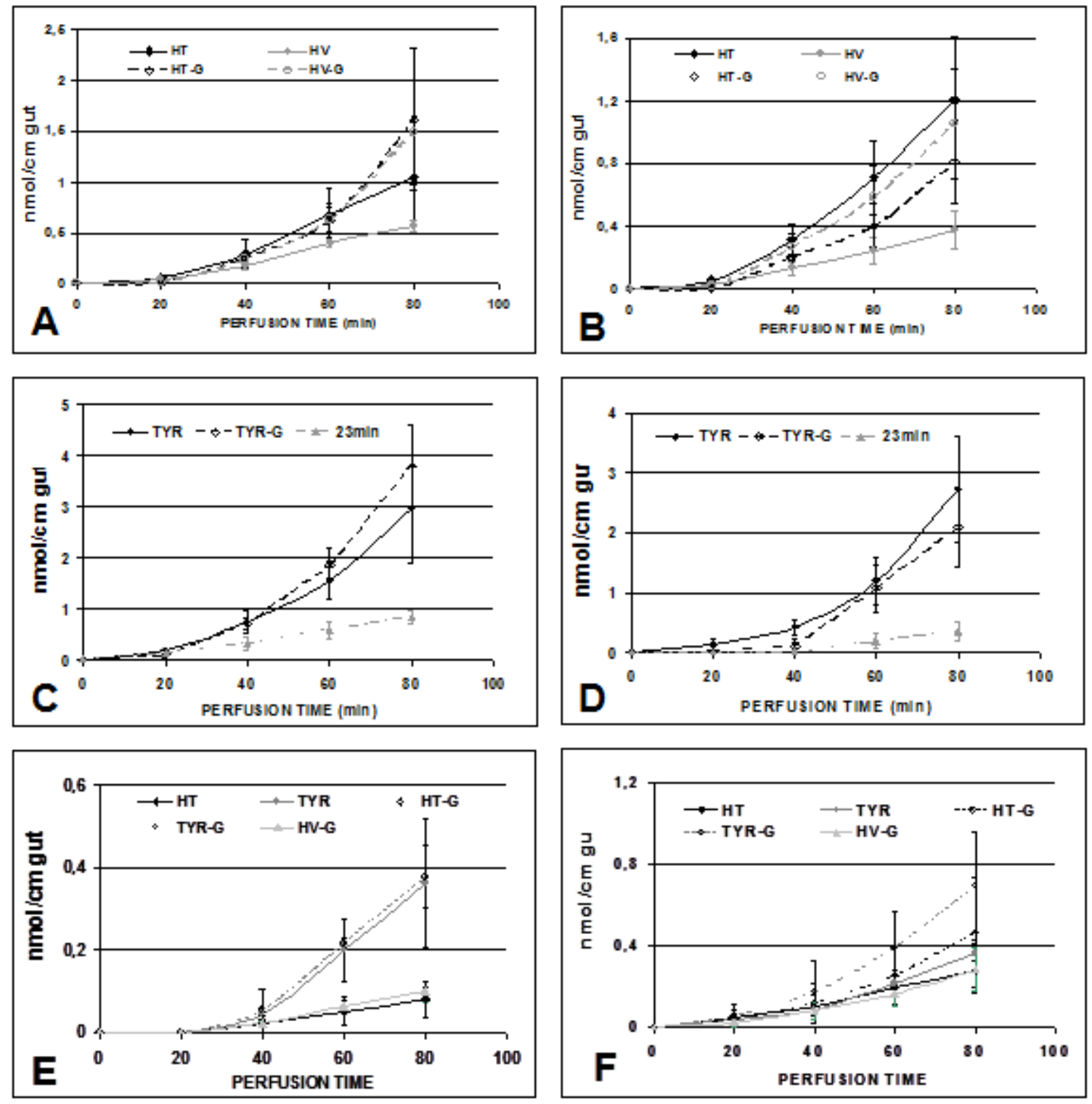
Figure 3
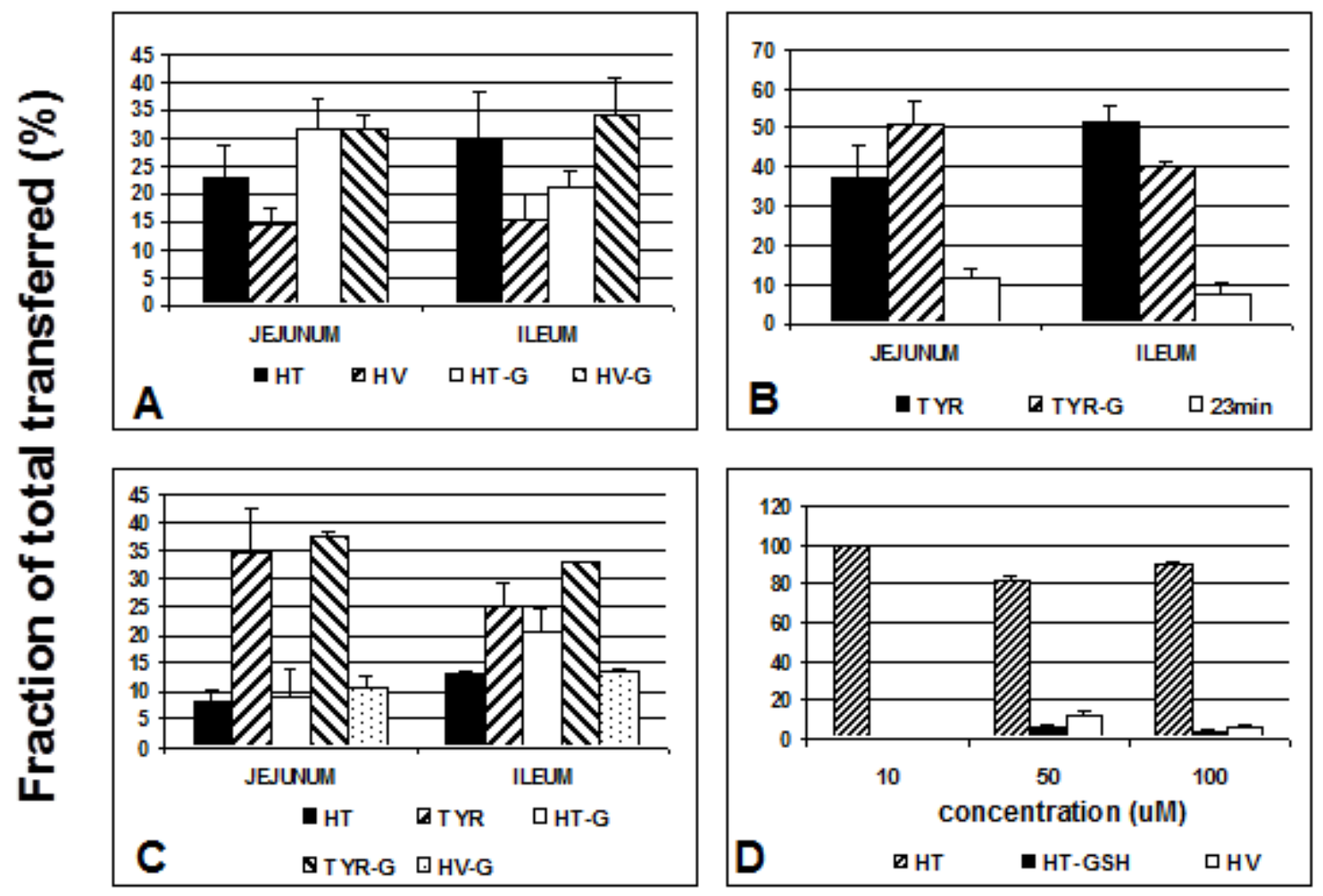
Figure 4

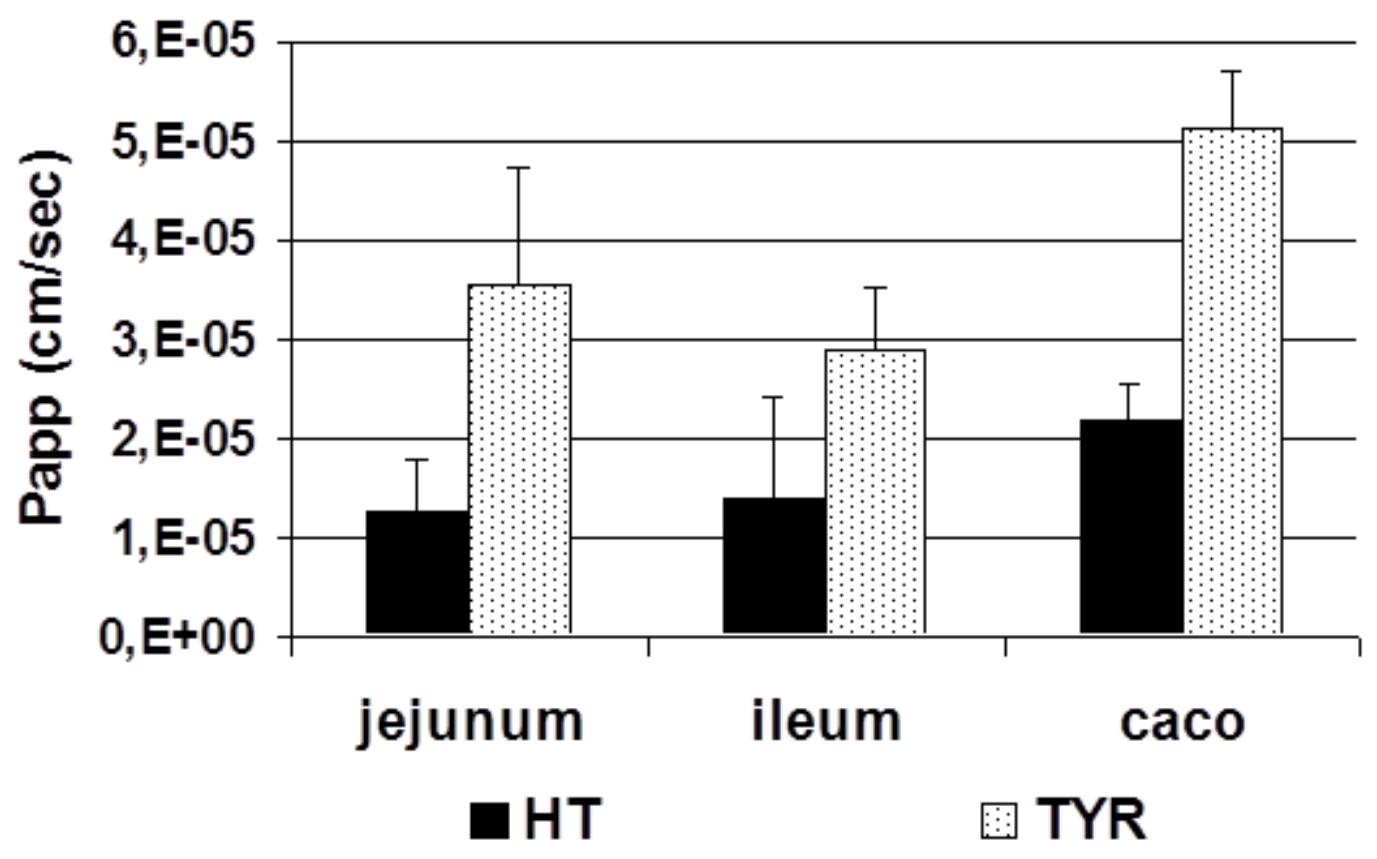


Figure 5
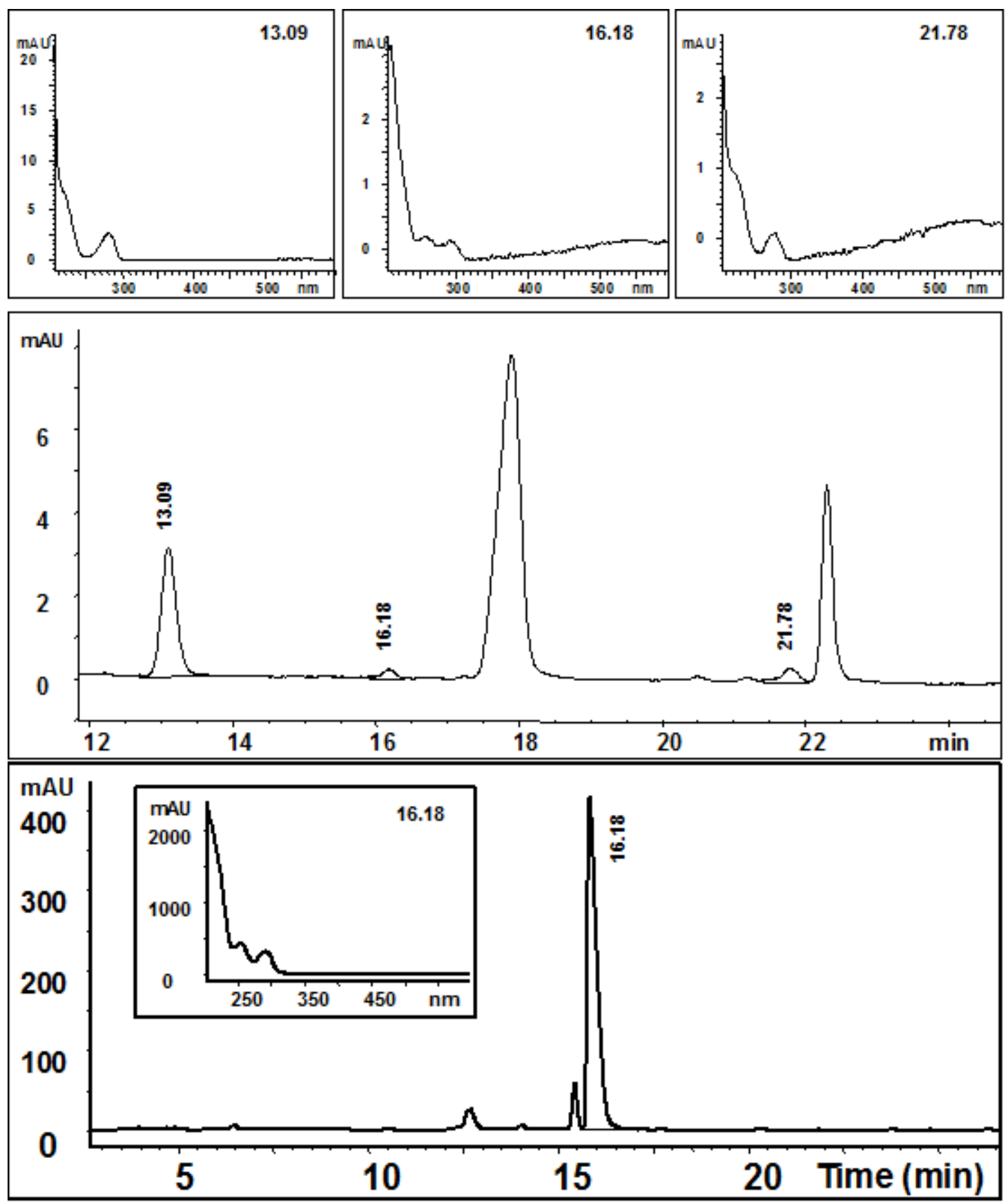
Figure 6

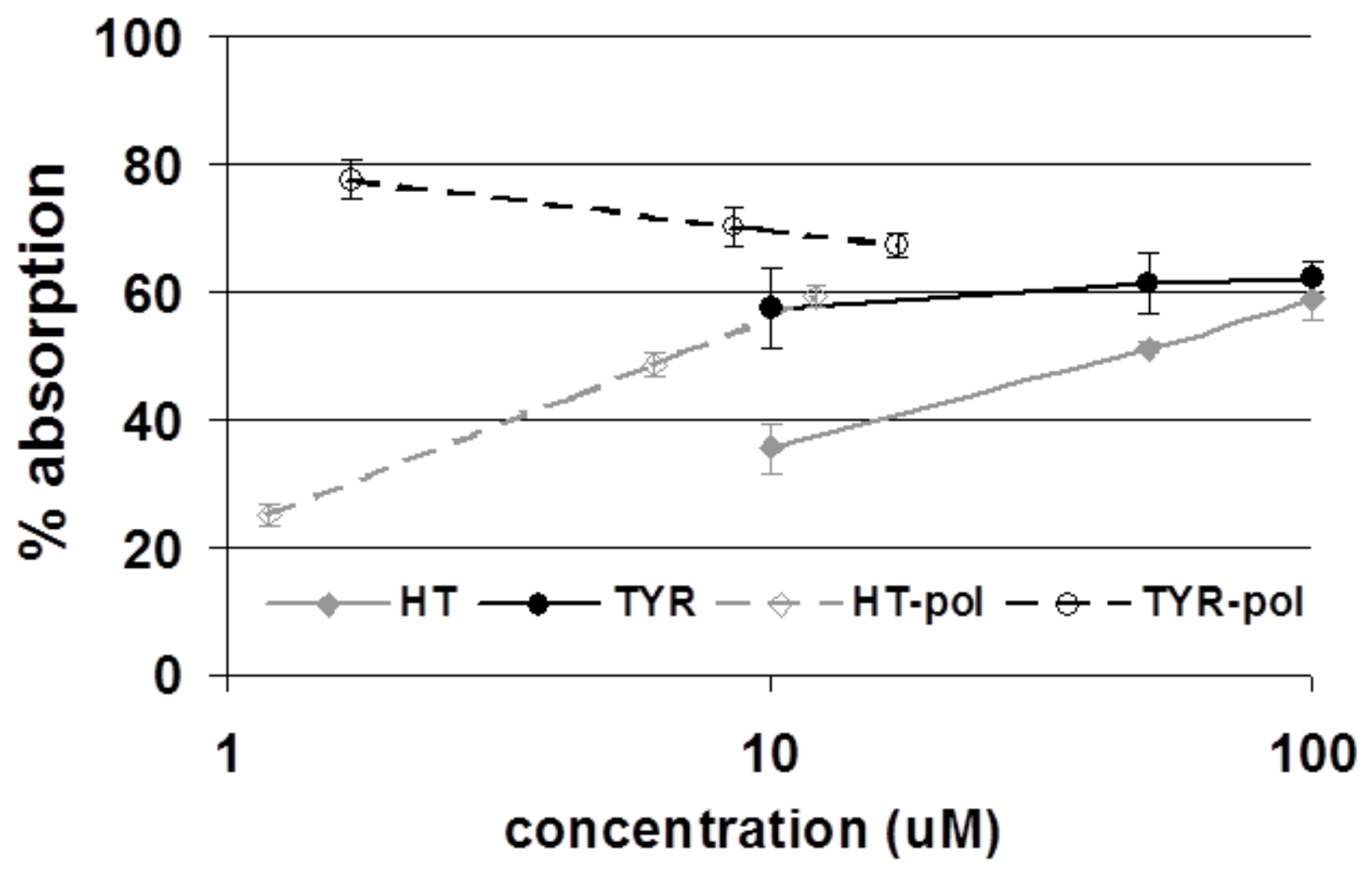


Figure 7

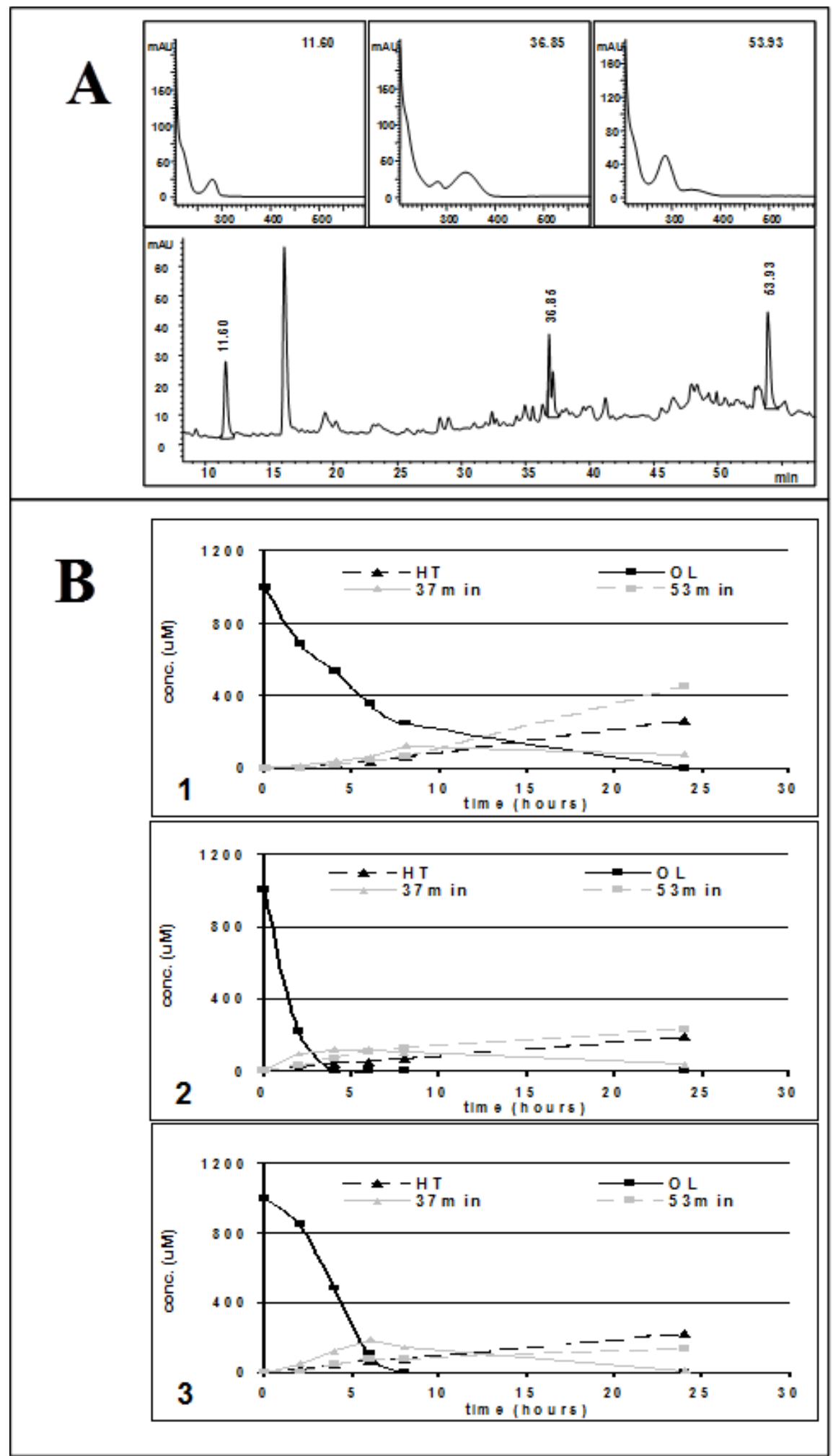


Figure Legends

Figure 1. HPLC chromatogram obtained from a control (panel A) and a sample (panel B) after $2 \mathrm{~h}$ incubation in $\mathrm{HCl}$. Detection was performed at $280 \mathrm{~nm}$. Amounts of hydroxytyrosol and tyrosol obtained after hydrolytic treatment of olive oil polyphenolic fraction over time (panel C). Data are plotted as the mean \pm S.E.M. $(n=3)$ and all values are significantly different respect to the control $(p<0.001)$.

Figure 2. Cumulative absorption of olive oil polyphenols and their metabolites over $80 \mathrm{~min}$ through isolated rat jejunum ( $A, C$ and $E)$ and ileum $(B, D$ and $F)$ after perfusion with hydroxytyrosol $(A$, $B)$, tyrosol $(C, D)$ and polyphenolic fraction $(E, F)$. Data are plotted as the mean \pm S.E.M. of three separate experiments.

Figure 3. Relative amount of hydroxytyrosol (A, D), tyrosol (B) and polyphenols (C) and their conjugates and metabolites after perfusion through the isolated rat jejunum and ileum $(A, B, C)$ and after $2 \mathrm{~h}$ incubation in Caco-2 cells (D). Data are plotted as the mean \pm S.E.M. of three separate experiments.

Figure 4. Apparent permeability coefficients (Papp) of hydroxytyrosol and tyrosol in jejunum, ileum and Caco-2 monolayers. Data are plotted as the mean \pm S.E.M. $(n=3)$ and all values are significantly higher than $1 * 10^{-6}(p<0.001)$.

Figure 5. HPLC chromatogram with photodiode array detection $(200-600 \mathrm{~nm})$ of the cell culture

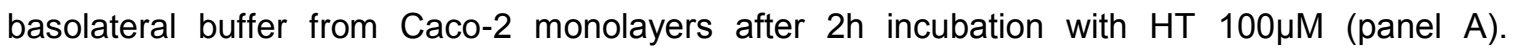
Detection was performed at $280 \mathrm{~nm}$. Peaks at $13.09 \mathrm{~min}, 16.18 \mathrm{~min}$ and $21.78 \mathrm{~min}$ RT are hydroxytyrosol, his glutathionyl-conjugate and homovanillyl alcohol respectively. HPLC 
chromatogram with photodiode array detection (200-600nm) of the HT-GSH conjugate (panel B) obtained after HT incubation in presence of GSH and tyrosinase.

Figure 6. Absorption of hydroxytyrosol and tyrosol in Caco-2 cells after $2 \mathrm{~h}$ incubation with the standard compounds (HT and TYR) and with the polyphenolic fraction (HT-pol and TYR-pol). Data are plotted as the mean \pm S.E.M. of three separate experiments.

Figure 7. Example HPLC chromatograms with photodiode array detection (200-600nm) representing the colonic biotransformation of oleuropein after $8 \mathrm{~h}$ fermentation (panel A). Detection was performed at $280 \mathrm{~nm}$. Time-fermentation profiles of oleuropein and its metabolites (panel B) in three individual donors $(1,2,3)$. 